\title{
Proposal for Preventing Motor Vehicle Accident Related to Drowsy Driving in Patients With Narcolepsy
}

\author{
Jae Wook Cho ${ }^{1}$, Jun-Sang Sunwoo ${ }^{2}$, Soo Hwan Yim ${ }^{3}$, Daeyoung Kim ${ }^{4}$, Dae Lim Koo ${ }^{5}$, Hee-Jin Im ${ }^{6}$, \\ Hyeyun Kim ${ }^{7}$, Kyung Min Kim ${ }^{8}$, Kwang IK Yang ${ }^{9}$; On behalf of the Korean Sleep Research Society \\ ${ }^{1}$ Department of Neurology, Pusan National University Yangsan Hospital, Pusan National University College of Medicine, Yangsan, \\ ${ }^{2}$ Department of Neurosurgery, Seoul National University Hospital, Seoul, \\ ${ }^{3}$ Department of Neurology, Gangneung Asan Hospital, Ulsan University College of Medicine, Gangneung, \\ ${ }^{4}$ Department of Neurology, Chungnam National University Hospital, Chungnam National University College of Medicine, Daejeon, \\ ${ }^{5}$ Department of Neurology, Seoul Metropolitan Government Seoul National University Boramae Medical Center, \\ Seoul National University College of Medicine, Seoul, \\ ${ }^{6}$ Department of Neurology, Hallym University Dongtan Sacred Heart Hospital, College of Medicine, Hallym University, Hwaseong, \\ ${ }^{7}$ Department of Neurology, International St. Mary's Hospital, Catholic Kwandong University College of Medicine, Incheon, \\ ${ }^{8}$ Department of Neurology, Severance Hospital, Yonsei University College of Medicine, Seoul, \\ ${ }^{9}$ Sleep Disorders Center, Department of Neurology, Soonchunhyang University Cheonan Hospital, \\ Soonchunhyang University College of Medicine, Cheonan, Korea
}

\section{기면병 환자의 졸음운전 관련 교통사고 예방을 위한 제안}

조재욱 ${ }^{1}$, 선우준상 ${ }^{2}$, 임수환 ${ }^{3}$, 김대영 ${ }^{4}$, 구대림 ${ }^{5}$, 임희진 ${ }^{6}$, 김혜윤 ${ }^{7}$, 김경민 $^{8}$, 양광익 ${ }^{9}$ 대한수면연구학회

${ }^{1}$ 부산대학교 의과대학 양산부산대병원 신경과학교실, ${ }^{2}$ 서울대학교병원 신경외과, ${ }^{3}$ 울산대학교 의과대학 강릉아산병원 신경과학교실,

${ }^{4}$ 충남대학교 의과대학 충남대학교병원 신경과학교실, ${ }^{5}$ 서울대학교 의과대학 서울특별시보라매병원 신경과학교실,

${ }^{6}$ 한림대학교 의과대학 동탄성심병원 신경과학교실, ${ }^{7}$ 가톨릭관동대학교 의과대학 국제성모병원 신경과학교실,

8연세대학교 의과대학 세브란스병원 신경과학교실, ${ }^{9}$ 순천향대학교 의과대학 천안병원 수면장애센터 신경과학교실

Received November 23, 202

Revised December 19, 2021

Accepted December 21, 202

Address for correspondence

Kwang IK Yang, MD, PhD

Sleep Disorders Center,

Department of Neurology,

Soonchunhyang University

Cheonan Hospital,

Soonchunhyang University

College of Medicine,

31 Soonchunhyang 6-gil,

Dongnam-gu,

Cheonan 31151, Korea

Tel: +82-41-570-2290

Fax: +82-41-592-3810

E-mail: neurofan@schmc.ac.kr
Narcolepsy is a chronic sleep disorder characterized by irresistible sleep attacks, hypersomnolence, cataplexy (sudden loss of muscle tone provoked by emotion), and sleep paralysis. Individuals with narcolepsy are at a high risk of experiencing sleepiness while driving leading to road traffic accidents. To prevent such accidents, some countries have regulations for commercial and noncommercial drivers with narcolepsy. Evaluating sleepiness is essential. Therefore, several subjective reports and objective tests were used to predict the possibility of car crashes or near-misses. Brain stimulants are effective in treating narcolepsy and can reduce daytime sleepiness in these patients. However, no guideline has been established for the driving safety of patients with narcolepsy in Korea. The Korean Sleep Research Society has prepared this proposal for preventing motor vehicle accidents caused by drowsy driving in patients with narcolepsy.

J Sleep Med 2021;18(3):137-144

Keywords: Excessive daytime sleepiness; Traffic accidents; Narcolepsy; Automobile driving.

\section{서 론}

졸음운전은 국내외 교통사고 발생의 흔한 원인 중 하나 다. ${ }^{1,2}$ 좋지 않은 수면 위생, 즉 불규칙하거나 부족한 수면 시
간을 가진 상태로 운전하다가 졸리는 경우가 가장 많지만, ${ }^{3}$ 운전을 방해하는 수면장애 때문에 피로감과 졸음운전이 발생 하는 경우도 있다. 이러한 대표적인 수면 질환으로는 폐쇄수 면무호흡, 불면증 등이 있으며 그 이외에도 과다졸림의 중추

This is an Open Access article distributed under the terms of the Creative Commons Attribution Non-Commercial License (https://creativecommons.org/licenses/bync/4.0) which permits unrestricted non-commercial use, distribution, and reproduction in any medium, provided the original work is properly cited. 
장애(central disorders of hypersomnolence)로 분류되는 기 면병, 하루수면주기의 불규칙성 혹은 위상(phase)의 이상으 로 나타나는 하루주기리듬수면장애(circadian rhythm sleepwake disorders) 등이 있다. 졸음운전으로 인한 인명피해와 사회적 비용 낭비가 우려되지만 국내에서는 졸음운전의 기 초 자료가 부족하여 현황을 파악하고 대책을 제시하는데 어 려움이 있다. ${ }^{4}$ 특히 각종 수면장애로 인한 졸음운전에 대한 관심이 더 절실하다.

과다졸림의 중추장애 중 대표적인 기면병은 각성과 연관 된 신경전달물질인 히포크레틴(hypocretin)을 분비하는 신경 세포 손상을 받아 발병하는 것으로 주간과다졸림, 탈력발작, 입면 시 환각, 수면 마비를 특징으로 하는 만성 신경계 수면 질환이다. ${ }^{5}$ 특히 억제할 수 없는 졸음으로 갑자기 잠에 빠지 기도 하고 낮잠을 자주 잔다. 유병률이 전체 인구의 $0.05 \%$ 정도인 드문 질환으로 크게 1형과 2형으로 나눌 수 있는데, 기면병 1형은 히포크레틴 뉴런이 90\%이상 파괴되어 뇌척수 액 히포크레틴 농도가 $110 \mathrm{pg} / \mathrm{mL}$ 이하인 특징이 있다. 주간 과다졸림과 더불어 여러가지 렘수면과 관련된 증상이 갑자 기 나타날 수 있다. 웃거나 즐거운 감정이 근긴장도(muscle tone)를 감소시켜 근육의 힘이 빠지는 탈력발작 증상이 동반 되기도 한다. 기면병 2형은 정확한 병태생리는 아직 잘 모르 지만 주간과다졸림이 뚜렷한 증상이다. 기면병 환자들은 낮 에 심한 졸음을 느끼지만 짧은 낮잠(15분 이내)으로도 각성 을 일정기간 회복하는 것이 특징이다.

본 종설에서는 지난 호 종설 ${ }^{1}$ 에 이어, 기면병 환자들의 졸 음운전에 대해 고찰하고 교통사고를 줄이기 위한 방안을 제 안하고자 한다.

\section{본 론}

\section{기면병과 교통사고}

기면병 환자는 수면과 각성 전환이 불안정하게 반복되는 병의 특성상 하루 중 언제라도 졸림이 발생할 수 있어, ${ }^{6}$ 졸음 운전의 위험도가 높고 차 사고 빈도가 더 높다고 알려져 있 는데, ${ }^{78}$ Aldrich ${ }^{9}$ 는 졸음과 관련된 자동차 사고 빈도가 기면병 환자에게서 일반인에 비해 3-4배 더 높다고 보고하였다(Table 1). 폐쇄수면무호흡 환자와 비교하면 치료받지 않은 기 면병의 경우 졸음운전의 위험성이 높지만, 기면병은 유병률 이 높지 않은 드문 질환이므로 절대적 졸음운전 사고 수치는 수면무호흡이 더 높다. ${ }^{9}$ 주간과다졸림증은 기면병 환자의 삶 에 가장 큰 영향을 미치는 증상으로, 성인 기면병 환자의 $66 \%$ 가 운전 도중 잠에 빠진 적이 있고, $67 \%$ 가 졸음운전 때문에 사고가 나거나 날 뻔한 아차사고(near-miss accident)를 경
험한 적이 있다고 답하였다. ${ }^{10}$ 또한, $29 \%$ 의 환자는 운전 도중 탈력발작을 경험하였고, $12 \%$ 는 운전 중 수면마비를 겪었다 고 하여, 졸림 외의 증상으로도 교통사고의 위험성이 높다. 기면병 1형과 2형으로 분류하여 졸음운전의 발생률을 비교 한 연구는 아직 없다.

직업적으로 장거리를 운전해야 하는 시외버스 혹은 화물차 운전의 경우 졸림을 더 자주 경험할 수 있는 환경이다. 아직 국내 연구는 없으나 외국의 경우 장시간의 운전, 교대근무, 위 험물질 적재, 버스나 화물차 등 대형 차량 등 복합적인 요인 들이 시너지 효과를 일으켜 사고의 위험이 높고 사고 시 피해 규모가 더 커질 위험이 있다. ${ }^{11-13}$ 과도한 졸림을 가지는 기면 병 환자의 경우 상대적으로 더 위험할 수 있음을 짐작할 수 있 다. 이러한 이유로 2010년 미국 Federal Motor Carrier Safety Administration's Medical Review Board에서는 아무리 치료 를 잘 받고 있는 상태라 하더라도 기면병 환자는 사업용 차량 (commercial vehicle) 운전에 부적격이라는 결론을 내렸다. ${ }^{14}$

\section{졸음운전 예방을 위한 기면병 치료의 효과}

기면병은 아직 완치를 기대하기 힘든 난치성 질환으로 분 류되어 있으며 적절한 약물 처방과 생활습관교정으로 정상 적인 일상생활을 영위할 수 있도록 치료하는 것을 목표로 한 다. 기면병으로 진단받으면 모다피닐(modafinil), 아모다피닐 (armodafinil), 메틸페니데이트(methylphenidate) 등의 뇌자 극제를 복용함으로써 주간졸림을 예방할 수 있다. ${ }^{5}$ 외국에서 는 gamma-hydroxybutyrate acid 혹은 피톨리산트(pitolisant) 등의 약물도 사용하지만 아직 국내에서는 처방이 불가능하다. 일부 환자에게는 규칙적인 낮잠, 특히 오후 1-2시에 취하는 짧은 낮잠이 1-3시간 정도의 각성을 유지하는 효과가 있다. ${ }^{15}$

기면병 환자들의 치료가 졸지 않고 집중해서 운전할 수 있 는 능력을 향상시킬 수 있는지에 대한 연구는 아직 많지 않 다. 하지만 소규모로 이루어진 몇몇 연구들은 기면병 혹은 특 발수면과다증 환자들이 모다피닐을 복용했을 때 운전사고를 줄일 수 있다는 결과를 보여주었다. 모다피닐 $400 \mathrm{mg}$ 을 5일 복용하였을 때 그렇지 않은 군에 비해 각성유지검사(Maintenance of Wakefulness Test, MWT)에서 수면잠복기가 유 의하게 늘었으며 운전능력도 향상되었다. ${ }^{16} 10$ 명의 기면병 환자와 17 명의 특발수면과다증 환자들을 27 명의 대조군과 비교한 연구에서도 모다피닐을 복용한 과다졸림의 중추장애 환자들은 약물을 복용하지 않은 환자들보다 MWT에서 더 긴 평균 수면잠복기와 더 나은 운전수행능력을 보였다. ${ }^{17}$ 흥 미롭게도, 약물을 복용한 과다졸림의 중추장애 환자들은 여 전히 정상 대조군보다는 짧은 수면잠복기를 가졌지만 운전

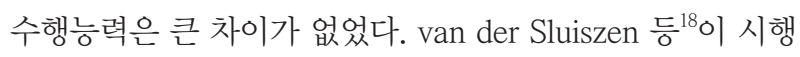


Cho JW et al.

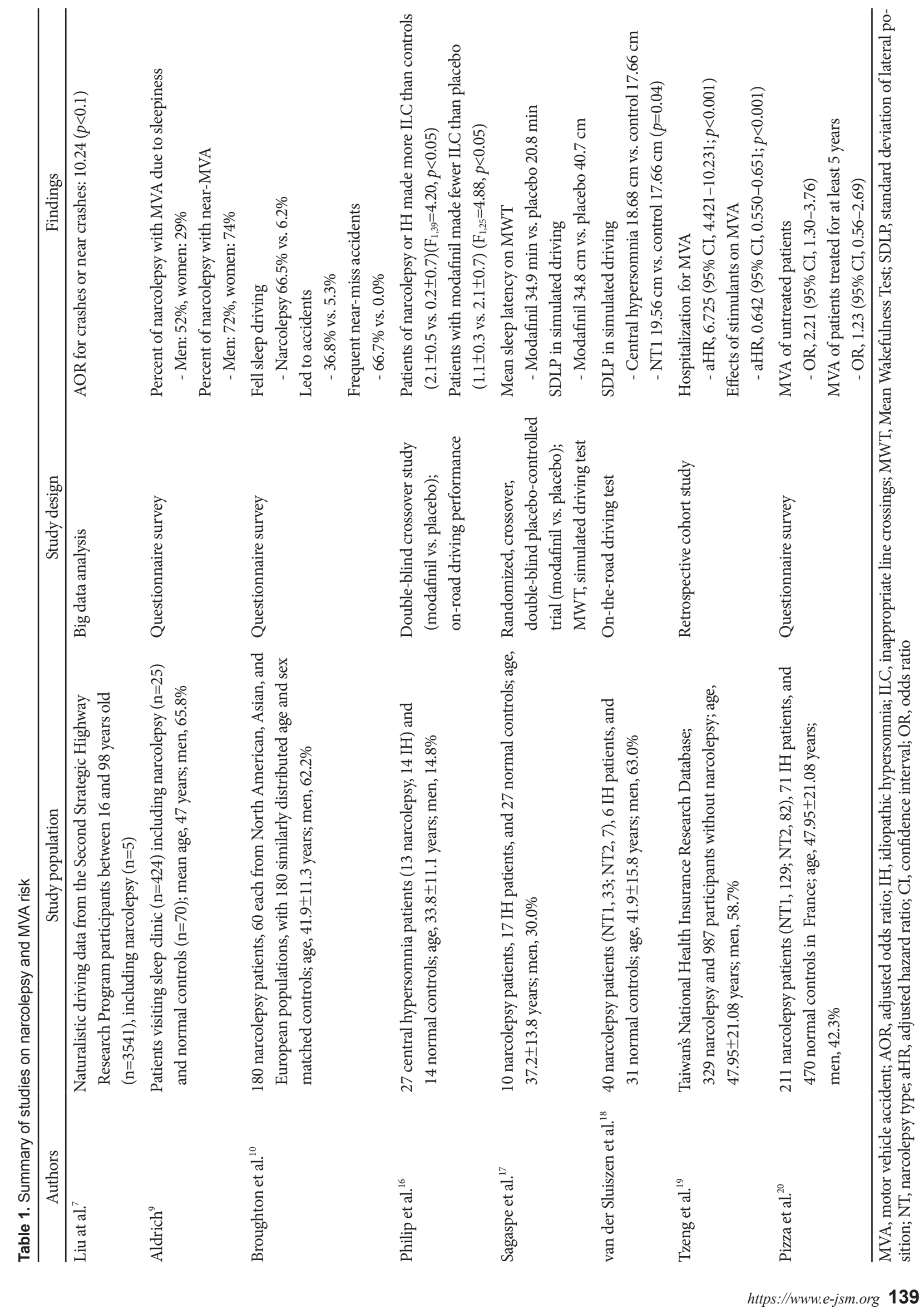


한 최근 연구에서는 약물복용으로 치료중인 과다졸림의 중 추장애 환자가 실제 도로 위에서 시행한 on-the-road 운전 수행능력 연구에서 정상 대조군에 비해 평균적으로는 열등 하지 않은 운전능력을 보였다. 그러나 그 중 기면병 환자들은 개인 편차가 매우 큰 결과값을 나타냈다. 특히 기면병 1형 환 자의 운전 능력이 상대적으로 더 많이 떨어져, 33 명의 환자 중 6 명이 운전 중 졸림으로 인해 1 시간 길이의 운전 테스트 를 완료하지 못하였다. 이는 아무리 약물치료 중이더라도 기 면병 환자 중 일부는 장시간 운전이 적합하지 않을 수 있다 는 것을 의미한다. 교통사고로 입원한 기면병 환자를 대상으 로 한 연구에서는 모다피닐과 메틸페니데이트를 복용하는 환자가 사고의 위험성이 상대적으로 낮았으며, ${ }^{19}$ 약물 치료 중인 환자는 정상 대조군과 비교해 사고 횟수에 큰 차이가 없다는 연구결과도 있었다. ${ }^{20}$ 이와 같은 연구결과들은 적절한 약물치료가 분명 졸음운전 교통사고를 감소시킬 수 있음을 보여준다. 기면병의 졸림 치료로 사용되는 기타 약물의 효과 에 대한 단독 연구는 아직 없다.

일부 기면병 환자들은 낮잠을 취함으로써 각성 효과를 얻 을 수 있으므로 운전에 도움이 될 것으로 기대할 수 있다. ${ }^{21,22}$ 평소 규칙적인 스케줄로 낮잠을 자는 환자라면 운전시에도 시각에 맞춰 낮잠을 자는 것이 도움이 될 것이다. 장거리 운 전 시에도 중간중간 낮잠을 취하면 각성 효과가 있을 것이다. 낮잠 길이는 $15-20$ 분 정도가 적당하며 더 길게 낮잠을 취할 경우 오히려 sleep inertia로 인해 무기력해질 수 있다. ${ }^{23}$ 그러 나 이러한 낮잠이 기면병 환자의 운전 능력을 얼마나 호전 시키는지에 대한 연구는 아직 없다.

카페인 섭취는 일반인을 대상으로 한 연구에서는 일시적 인 각성효과와 함께 운전능력을 향상시킬수 있으리라 기대 할 수 있으나 그 효과는 매우 짧고 기면병 환자의 졸음운전에 대해서는 아직 연구된 바가 없다. 그 외 껌씹기, 음악 틀기, 창문을 열어 바람을 쐬기, 옆사람과 대화 등 일반적으로 졸 음운전 예방 방법으로 알려진 행위들에 대해서도 졸음 운전 방지에 얼마나 효과가 있는지 증거가 부족하다.

\section{졸림 정도를 측정하는 방법}

진단을 위해서는 수면에 대한 병력 청취 후 야간 수면다 원검사와 다중수면잠복기검사(Multiple Sleep Latency Test, MSLT)를 시행한다. MSLT는 일반적으로 야간수면다원검 사 다음날 낮에 시행하는데 4-5회 낮잠을 자는 상황에서 각 검사 시작부터 잠이 드는 수면잠복기와 렘수면이 나타나는 시간을 측정한다. MSLT에서 평균 수면잠복기가 8분 이하이 면서, 렘수면이 입면 후 15 분 이내에 관찰되는 입면기렘수면 (sleep onset rapid eye movement periods, SOREMPs)이
2 회 이상 관찰되는 경우 기면병의 진단 범위에 해당한다. 뇌 척수액 히포크레틴 농도가 낮음을 확인할 수도 있지만 아직 국내에서는 검사가 불가능하다.

아직까지 기면병 환자의 교통사고 방지를 위해 졸림 정도 를 측정하는 가이드라인은 없다. ${ }^{8}$ 운전 전에 적절한 졸림 평 가도구를 활용해서 운전자가 자신의 졸림 정도를 스스로 평 가할 수 있고, 졸음운전의 구체적 증상이 있음을 알 수 있다 면, 졸음운전 사고의 위험을 줄일 수 있을 것이다. ${ }^{24}$

스탠포드졸림척도(Stanford Sleepiness Scale)는 주관적인 졸림 척도로 현재 시점의 졸림 정도를 1점(최고로 명료함)부 터 7점(최고로 졸림)으로 구분하며, 3점 이상이면 졸림으로 인해 업무 수행능력이 떨어질 수 있음을 의미한다. ${ }^{25}$ 주관적 으로 자신의 졸림 정도를 평가하기 위해 널리 사용되는 척도 는 엡워스졸림척도(Epworth Sleepiness Scale, ESS)다. ${ }^{26}$ 여 러가지 상황에서 얼마나 졸리는지를 평가하는 방법인데 쉽 고 간단하게 측정할 수 있다는 장점이 있다. 그러나 이 점수 가 교통사고의 위험성과 연관성이 있는지에 대해서는 연구 마다 다른 결과를 보였다. 일부 연구에서는 교통사고 위험과 $\mathrm{ESS}$ 의 높은 점수가 유의한 상관관계를 보였으나, 27,28 그렇지 못한 경우도 있었다. ${ }^{17}$ 그리고 운전자는 졸림으로 인해 본인 의 운전 능력이 떨어짐을 자각하지 못하는 경우가 많다..$^{29}$

따라서 정확한 판단을 위해서는 객관적인 방법이 필요하 다. 임상적으로 안전운전과 관련해서 많이 사용되는 객관적 졸림 측정 방법은 $\mathrm{MWT}$ 검사다. ${ }^{30}$ 환자는 어두운 방에서 잠 들지 않고 조용히 앉아 각성을 유지해야 하는데, 1 세션 당 최 대 40분 동안 시행하며 검사 시작부터 잠이 들 때까지의 수 면잠복기를 측정한다. 총 4세션을 시행하여 계산한 평균수 면잠복기가 8분 이하면 비정상으로 졸린 상태로 간주하고, 40 분 이상일 경우 정상이다. 하지만 MWT 역시 짧은 잠복기 가 교통사고 위험성이 더 높음을 예측할 수 있는지는 연구 마다 결과가 달라 아직 추가적인 연구가 필요하다. ${ }^{30}$ Ingram 등은 여러 연구들을 종합하여, 기면병 환자들의 경우 MWT 검사에서 운전에 필요한 평균수면잠복기를 최소 30 분 이상으 로 제안하였다. 얼마나 빨리 잠이 드는지를 확인하는 MSLT 의 경우 운전 능력과의 연관성은 없는 것으로 알려져 있다. 17,20

운전 능력을 테스트하는 방법은 여러가지가 있다. 컴퓨터 모니터에 단순하게 보이는 차와 도로의 그래픽을 보며 장애 물이 나타났을 때 피하기 위해 키보드를 누르는 형태로 반 응시간(reaction time)을 측정하는 원시적인 방법부터, 실제 도로에서 주행하면서 차선을 얼마나 자주 침범하는지 등을 확인하는 테스트까지 다양하다. ${ }^{12}$ 시뮬레이션 운전을 이용한 과거 여러 연구에서 기면병 환자들은 정상군에 비해 낮은 수행점수 보였다. 하지만 이러한 연구들은 대부분 연구용으 
로 세팅 된 장소에서 운전하거나 시뮬레이션 장치에서 평가 한 것들이었고 환자들이 실생활에서 운전하는 환경을 반영 한다고 할 수 없기에, 이 결과값이 실제 사고 위험성을 예측 한다고 단정하기는 어렵다. 또한, 모든 환자 혹은 운전자를 대상으로 시행하는 것도 실용적이지 않다.

운전 중 뇌파, 안구운동, 근전도, 심전도 등 생리적 신호 (physiological signals)를 측정하여 잠이 드는 것을 확인하는 방법도 연구되었다. Watling 등 32 은 메타분석연구를 통해 단 일 신호만 분석하기보다 여러 신호를 조합하여 졸림을 판단 하는 것이 졸림 판별의 민감도와 특이도를 높일 수 있음을 보여주었다. 아직 소규모 연구들만 진행이 된 단계지만 추후 다양한 방법으로 정확도를 높일 수 있다면 실제 운전 중 졸 림을 판단할 수 있는 방법으로 유용할 것이다.

과거 졸음운전으로 인한 사고 혹은 아차사고의 이력을 문 진으로 청취하는 것도 도움이 된다. 우리나라보다 청소년들 의 운전이 더욱 보편화된 미국에서는 $88 \%$ 의 의사가 청소년 기면병 환자를 진료할 때마다 운전 가능성에 대해 평가를 한 다고 하였는데, 졸음운전으로 사고가 났거나 날 뺀했던 적이 있었는지, 환자 본인 혹은 동승자에 의해 졸음운전 혹은 탈 력발작이 목격된 적이 있는지 등의 질문이 교통사고 예측에 매우 중요하다고 답하였다. ${ }^{31}$ 이 연구에서는 ESS 등 주관적 인 주간졸림 척도도 운전 가능 평가에 중요하다고 생각하였 으나 MSLT나 MWT와 같이 객관적으로 졸림을 측정하는 수면검사는 상대적으로 중요도를 낮게 평가하였다.

하지만 아직 어떤 자가측정이나 검사도 졸음운전을 예측하 기에는 한계가 있다. $\mathrm{ESS}$ 는 주관적으로 응답하는 설문 도구 이므로 고용과 운전면허 취득과 같은 이해관계와 관련된 상 황들이 답변에 영향을 줄 수 있다. ${ }^{33}$ 수면다원검사와 MSLT 혹은 MWT 검사는 기면병 환자의 주간과다졸림 심한 정도 를 평가할 객관적 증거를 제시할 수 있으나 단 하루의 검사 라는 단점이 있으며, 검사 결과가 자신의 직업에 영향을 준 다면 그러한 동기(motivation)가 검사결과에 영향을 줄 수 있다. 무엇보다도, 검사의 결과가 졸음운전 사고의 위험성과 직접적으로 연관이 있는지에 대한 증거가 아직 부족하다. ${ }^{9,34}$ 따라서 MWT의 결과만으로 교통사고 발생 가능성을 판단 해서는 안 된다. ${ }^{35}$ 액티그래피를 이용한 수면시간 측정도 졸 음운전을 예방하기 위한 평가 도구로 사용하기엔 적합하지 않다. 탈력발작의 경우 증상의 심한 정도를 객관적으로 나타 내는 지표가 아직 없으며, 마찬가지로 병력 청취 외엔 환자 의 교통사고 위험을 예측할 수 있는 방법이 없다. ${ }^{8}$

\section{기면병 환자의 운전에 대한 다른 나라의 권고사항}

개인마다 수면의 내재적 요인(예: 아침형 저녁형, 적절한
하루 수면시간 등)이 다르고, 수면부족이나 변화된 수면리듬 상황에 대한 적응 정도가 다르다. 기면병 증상의 심한 정도 와 졸림이 졸음운전에 미치는 영향도 개인차가 있으므로, 환 자 전체를 대상으로 공통된 규칙을 정하기보다는 표현형을 좀 더 세분화하고 졸음운전 고위험군을 정의하고 선별하는 과정이 필요하다. ${ }^{36}$ 또한, 정책적으로 이를 일반 운전과 직업 운전에 어떻게 적용하여 사고를 예방할 수 있을지 논의가 필 요하다. 졸음운전의 가능성이 높은 기면병 환자는 운전면허 발급을 보류하는 등의 적극적 방안도 생각할 수 있다.

미국 일부 지역, 호주, 그리고 유럽 여러 나라에서 기면병 환자의 운전면허 발급에 제한을 두거나 조건을 거는 조치들 이 시행되었다(Supplementary Table 1 in the online-only Data Supplement). 예를 들어, 미국 캘리포니아에서는 기면 병 증상이 얼마나 오래 조절되는지에 따라 구분하여 제한적 인 면허를 발급하며, 의사가 운전하기 적합하지 않다고 판단 할 경우엔 면허가 정지된다. ${ }^{37}$ 미국 6개 주(California, Delaware, Nevada, New Jersey, Oregon, Pennsylvania)에서는 기면병을 비롯하여 운전에 영향을 줄 수 있는 환자 상태에 대해 미리 보고하도록 의무로 규정하고 있다. ${ }^{8}$ 캐나다에서는 수면발작(sleep attack)이나 탈력발작을 자주 경험하는 환자 는 어떠한 종류의 운전도 허용되지 않는다. 적절한 치료로 증상과 약물 부작용이 없는 상태가 최소 12 개월 이상이 되어 야 운전이 가능하다. ${ }^{38}$ 영국에서는 기면병으로 진단을 받으면 증상의 정도에 상관없이 즉시 Driver and Vehicle Licensing Agency (DVLA)에 알려야 하며,,$^{39}$ DVLA에서 수면전문의사 의 자문을 참고하여 운전 가능 여부를 결정한다. 기면병 환자 의 직업 운전면허(vocational license) 취득은 원칙적으로는 불가능하며, 일반 운전면허는 증상이 잘 조절된다는 의학적 소견을 첨부하였을 때만 한시적으로 발급받을 수 있다..$^{40}$ 네 덜란드 Transport Division of the Ministry of Transport에서 도 탈력발작을 동반한 기면병의 경우 버스, 트럭 등 사업용 차량 운전은 금지하고 있으며, 개인 차량의 경우 최소 2 개월 이상의 치료를 받고 운전이 가능하다는 수면 전문가의 리포 트를 근거로 한시적으로 가능한 면허를 발급한다. ${ }^{41}$ 호주에 서는 기면병 증상이 조절되지 않으면 모든 운전이 금지되며 특히 탈력발작이 있을 경우 사업용 차량 운전은 불가한 것으 로 간주한다. 적절한 치료로 수면 전문가의 자문을 얻어 운 전에 문제가 없다고 판단된 경우에만 개인 승용차 운전은 가 능하다. 사업용 차량의 경우 매년 건강 상태를 보고해야 하 며, 약물 복용을 규칙적으로 유지하고 최소 6개월 이상 과다 졸림 증상이 없으며, MWT에서 정상 범위의 수면 잠복기를 보인다는 조건부로 운전면허 발급이 가능하다. ${ }^{42}$ 뉴질랜드도 수면 전문가에 의한 적절한 처방으로 주간졸림증이나 탈력 
발작 증상이 잘 조절되는 경우에만 운전이 가능하며, 주간졸 림증이나 탈력발작이 심한 기면병 환자는 원칙적으로 사업 용 차량 운전은 허용하지 않는다. ${ }^{43}$

\section{기면병 환자의 운전에 대한 제안}

이러한 문헌고찰을 토대로 다음과 같이 제안한다.

\section{의사에게 제안}

- 주간졸림 환자 초진 시 교통사고가 난 적 있었는지, 교통 사고가 날 뻔한 아차사고가 있었는지 반드시 문진하고 재진 진료 때마다 문진으로 재확인한다.

- 졸음운전으로 교통사고가 났거나 날 뻔했던 기면병 환자 는 졸음운전 고위험군으로 분류하여 운전이 위험할 수 있음을 경고하고 가급적 운전을 자제할 것을 권유한다.

- 졸음운전 고위험군 환자가 부득이하게 운전해야 할 경 우에는 적절한 약물치료에도 불구하고 운전 중 졸림이 나 탈력발작이 갑자기 발생할 수 있음을 설명한다. 장거 리 운전 시엔 추가적인 약물복용을 고려하고 적절한 낮 잠 시간을 갖도록 교육한다.

- 기면병으로 약물 치료 중인 환자는 약물 순응도에 대한 문진이 필요하다. 재진 시 $\mathrm{ESS}$ 로 주간졸림의 정도를 확 인한다. 만약 주간졸림이 호전되지 않았거나 더 증가했 을 경우 졸음운전의 위험성을 더 자세하게 평가하고 치 료방법을 재고한다.

\section{환자에게 제안}

- 기면병이 의심되어 검사 받거나 기면병으로 진단받은 환자는 졸음운전에 대해 질문 받아야한다. 최근 졸림, 피 곤함, 판단착오 등으로 교통사고를 유발했거나 유발할 뼌한 적이 있는지 확인받아야 한다.

- 교통사고를 경험했거나 수면 증상으로 사고가 날 뺀했 던 경우라면 졸음운전 고위험 군으로 분류되어 운전이 위험할 수 있음을 이해한다.

- 운전 전 야간근무, 단당류 섭취, 졸음을 유발할 수 있는 약물 복용(ex. 항히스타민제, 안정제 등)을 피한다.

- 직업으로 장시간 운전을 요하는 일은 피한다(ex. 화물트 럭, 시외버스 등등)

- 기면병 환자가 부득이하게 장거리 운전을 해야 할 경우 에는 다음과 같은 방법을 고려해 볼 수 있다.

1. 의사에게 처방 받은 뇌자극제를 운전 30 분 전에 복용

2. 한번 운전할 시간을 1 시간 혹은 2 시간 등으로 제한하 여 규칙적인 간격으로 15-20분 정도 낮잠을 잘 것

3. 평소 규칙적으로 낮잠을 자는 스케줄이 있다면 해당
시간에는 반드시 휴게소나 심터에서 낮잠을 잘 것 4. 동행하는 일행이 있다면 운전을 교대로 할 것

\section{결 론}

기면병 졸음운전에 대한 문헌들을 고찰한 결과를 다음과 같이 정리할 수 있다.

- 기면병 환자는 주간과다졸림과 탈력발작 등의 증상으로 인해 교통사고를 유발할 가능성이 높으나 그 정도는 개 인차가 있다.

- 졸음운전 고위험군은 실제 졸음운전으로 교통사고를 경 험한 적이 있거나, 교통사고가 날 뻔한 환자다.

- ESS 등의 자가 졸림 측정이나 MWT, MSLT 등의 수면검 사 결과만으로 졸음운전의 가능성을 예측할 수는 없다.

- 기면병에 대한 적절한 약물 치료가 교통사고 위험성을 낮출 수 있다.

- 기면병 환자의 낮잠은 각성 유지 효과가 있어 사고를 줄 일 수 있을 것으로 기대된다. 하지만 아직 직접적인 근 거는 없다.

- 탈력발작 등 졸림 외 증상으로도 교통사고가 더 많이 발 생할 위험이 있으나 아직 구체적인 연구 결과가 없다.

- 적절한 진단과 치료, 환자와 보호자의 졸음운전과 수면 위생에 대한 교육을 통해 교통사고의 위험성을 낮출 수 있을 것으로 기대된다.

- 과거 교통사고의 이력이 없는 기면병 환자에게 운전면 허 발급을 제한하는 것은 아직 근거가 없다.

- 기면병 환자들의 직업 운전은 졸음운전으로 인한 사고 의 위험성이 높다.

기면병은 병적 증상으로 졸림이 갑자기 발생할 수 있으므 로 졸음운전으로 초래되는 교통사고의 책임은 운전자 본인 에게 있음을 인지하고, 졸음운전 예방을 위해 환자 본인이 노력해야 한다. 환자에 따라서는 기면병에 대한 적절한 약물 치료에도 불구하고 운전 중 졸림 증상이 예상치 못하게 발생 할 수 있으므로 이에 대한 경각심을 가지는 것도 필요하다.

정책적으로는 고위험군에 해당하는 기면병 환자는 운전 에 적합한지 아닌지를 판단하여 자격을 부여하는 방안을 수 면전문 의료진과 고민해 봄 직하다. 유럽 European Respiratory Society에서 테스크포스 팀을 만들어 폐쇄수면무호흡 환자의 운전면허 발급과 관련한 가이드라인을 정한 사례는 참고할 만하다. ${ }^{44}$

기면병 환자의 졸음운전 예방에 의료진, 환자, 그리고 사 회적 관심이 절실하게 필요하다. 


\section{Supplementary Materials}

The online-only Data Supplement is available with this article at https:// doi.org/10.13078/jsm.210025.

\section{Conflicts of Interest}

The authors have no potential conflicts of interest to disclose.

\section{ORCID iDs}

Jae Wook Cho Jun-Sang Sunwoo Soo Hwan Yim Daeyoung Kim Dae Lim Koo Hee-Jin Im Hyeyun Kim Kyung Min Kim Kwang IK Yang

https://orcid.org/0000-0002-2742-9136 https://orcid.org/0000-0001-8834-0568 https://orcid.org/0000-0003-2948-9244 https://orcid.org/0000-0001-9056-0017 https://orcid.org/0000-0001-6858-6093 https://orcid.org/0000-0002-8979-6521 https://orcid.org/0000-0002-8008-5539 https://orcid.org/0000-0002-0261-1687 https://orcid.org/0000-0001-6343-6520

\section{Author Contributions}

Conceptualization: Jae Wook Cho, Kwang IK Yang. Data curation: Daeyoung Kim. Formal analysis: Hee-Jin Im, Hyeyun Kim. Funding acquisition: Dae Lim Koo. Investigation: Daeyoung Kim, Dae Lim Koo. Methodology: Soo Hwan Yim, Jun-Sang Sunwoo. Project administration: Jae Wook Cho. Resources: Hee-Jin Im, Hyeyun Kim. Software: Jun-Sang Sunwoo, Daeyoung Kim. Supervision: Jae Wook Cho, Dae Lim Koo, Kwang IK Yang. Validation: Soo Hwan Yim, Kwang IK Yang. Visualization: Soo Hwan Yim, Kyung Min Kim. Writing-original draft: Jae Wook Cho. Writing_review \& editing: all authors.

\section{Funding Statement}

None.

\section{REFERENCES}

1. Yim SH, Cho JW, Sunwoo J, et al. Sleep disorders and risk of motor vehicle accident. J Sleep Med 2021;18:72-77. https://doi.org/10.13078/ jsm.210017.

2. Bioulac S, Micoulaud-Franchi JA, Arnaud M, et al. Risk of motor vehicle accidents related to sleepiness at the wheel: a systematic review and meta-analysis. Sleep 2017;40:zsx134. https://doi.org/10.1093/sleep/zsx134.

3. George CF. Sleep apnea, alertness, and motor vehicle crashes. Am J Respir Crit Care Med 2007;176:954-956. https://doi.org/10.1164/rccm.200605629PP.

4. Kim KT, Jung KY. Current status and proposal of drowsy driving. $J$ Sleep Med 2016;13:35-39. https://doi.org/10.13078/jsm.16007.

5. Kim H, Cho JW. Narcolepsy, how do we manage it? J Sleep Med 2020; 17:1-10. https://doi.org/10.13078/jsm.200006.

6. Valley V, Broughton R. Daytime performance deficits and physiological vigilance in untreated patients with narcolepsy-cataplexy compared to controls. Rev Electroencephalogr Neurophysiol Clin 1981;11:133-139. https://doi.org/10.1016/s0370-4475(81)80044-5.

7. Liu SY, Perez MA, Lau N. The impact of sleep disorders on driving safety-findings from the second strategic highway research program naturalistic driving study. Sleep 2018;41:zsy023. https://doi.org/10.1093/ sleep/zsy023.

8. McCall CA, Watson NF. Therapeutic strategies for mitigating driving risk in patients with narcolepsy. Ther Clin Risk Manag 2020;16:10991108. https://doi.org/10.2147/TCRM.S244714.

9. Aldrich MS. Automobile accidents in patients with sleep disorders. Sleep 1989;12:487-494. https://doi.org/10.1093/sleep/12.6.487.

10. Broughton R, Ghanem Q, Hishikawa Y, Sugita Y, Nevsimalova S, Roth B. Life effects of narcolepsy in 180 patients from North America, Asia and Europe compared to matched controls. Can J Neurol Sci 1981;8:299-304. https://doi.org/10.1017/s0317167100043419.

11. Philip P. Sleepiness of occupational drivers. Ind Health 2015;43:30-33. https://doi.org/10.2486/indhealth.43.30.

12. Dwarakanath A, Elliott MW. Assessment of sleepiness in drivers: current methodology and future possibilities. Sleep Med Clin 2019;14:441451. https://doi.org/10.1016/j.jsmc.2019.08.003.

13. Vennelle M, Engleman HM, Douglas NJ. Sleepiness and sleep-related accidents in commercial bus drivers. Sleep Breath 2010;14:39-42. https:// doi.org/10.1007/s11325-009-0277-z.

14. U.S. Department of Transportation. United States department of transportation federal motor carrier safety administration. Meeting summary [Internet]. Washington, DC: Federal Motor Carrier Safety Administration, 2010 [cited 2021 Nov 30]. Available from: https://www.fmcsa. dot.gov/sites/fmcsa.dot.gov/files/docs/Final_Jan_6_2010_MRB_Meeting_Summary.pdf.

15. Scammell TE. Treatment of narcolepsy in adults [Internet]. Riverwoods, IL: UpToDate, 2021 [cited 2021 Nov 30]. Available from: https://www. uptodate.com/contents/treatment-of-narcolepsy-in-adults.

16. Philip P, Chaufton C, Taillard J, et al. Modafinil improves real driving performance in patients with hypersomnia: a randomized double-blind placebo-controlled crossover clinical trial. Sleep 2014;37:483-487. https:// doi.org/10.5665/sleep.3480.

17. Sagaspe P, Micoulaud-Franchi JA, Coste O, et al. Maintenance of wakefulness test, real and simulated driving in patients with narcolepsy/hypersomnia. Sleep Med 2019;55:1-5. https://doi.org/10.1016/j. sleep.2018.02.009.

18. van der Sluiszen NNJJM, Urbanus B, Lammers GJ, Overeem S, Ramaekers JG, Vermeeren A. On-the-road driving performance of patients with central disorders of hypersomnolence. Traffic Inj Prev 2021; 22:120-126. https://doi.org/10.1080/15389588.2020.1862804.

19. Tzeng NS, Hsing SC, Chung CH, et al. The risk of hospitalization for motor vehicle accident injury in narcolepsy and the benefits of stimulant use: a nationwide cohort study in Taiwan. J Clin Sleep Med 2019;15: 881-889. https://doi.org/10.5664/jcsm.7842.

20. Pizza F, Jaussent I, Lopez R, et al. Car crashes and central disorders of hypersomnolence: a French study. PLoS One 2015;10:e0129386. https:// doi.org/10.1371/journal.pone.0129386.

21. Mullington J, Broughton R. Scheduled naps in the management of daytime sleepiness in narcolepsy-cataplexy. Sleep 1993;16:444-456. https:// doi.org/10.1093/sleep/16.5.444.

22. Garma L, Marchand F. Non-pharmacological approaches to the treatment of narcolepsy. Sleep 1994;17:S97-S102. https://doi.org/10.1093/ sleep/17.suppl_8.s97.

23. Dinges DF, Orne MT, Whitehouse WG, Orne EC. Temporal placement of a nap for alertness: contributions of circadian phase and prior wakefulness. Sleep 1987;10:313-329.

24. Williamson A, Friswell R, Olivier J, Grzebieta R. Are drivers aware of sleepiness and increasing crash risk while driving? Accid Anal Prev 2014;70:225-234. https://doi.org/10.1016/j.aap.2014.04.007.

25. Hoddes E, Zarcone V, Smythe H, Phillips R, Dement WC. Quantification of sleepiness: a new approach. Psychophysiology 1973;10:431-436. https://doi.org/10.1111/j.1469-8986.1973.tb00801.x.

26. Johns MW. A new method for measuring daytime sleepiness: the Epworth sleepiness scale. Sleep 1991;14:540-545. https://doi.org/10.1093/ sleep/14.6.540.

27. Philip P, Sagaspe P, Lagarde E, et al. Sleep disorders and accidental risk in a large group of regular registered highway drivers. Sleep Med 2010; 11:973-979. https://doi.org/10.1016/j.sleep.2010.07.010.

28. Howard ME, Desai AV, Grunstein RR, et al. Sleepiness, sleep-disordered breathing, and accident risk factors in commercial vehicle drivers. Am J Respir Crit Care Med 2004;170:1014-1021. https://doi.org/10.1164/ rccm.200312-1782OC.

29. Reyner LA, Horne JA. Falling asleep whilst driving: are drivers aware of prior sleepiness? Int J Legal Med 1998;111:120-123. https://doi. org/10.1007/s004140050131. 
30. Littner MR, Kushida C, Wise M, et al. Practice parameters for clinical use of the multiple sleep latency test and the maintenance of wakefulness test. Sleep 2005;28:113-121. https://doi.org/10.1093/sleep/28.1.113.

31. Ingram DG, Marciarille AM, Ehsan Z, Perry GV, Schneider T, AlShawwa B. Assessing readiness to drive in adolescents with narcolepsy: what are providers doing? Sleep Breath 2019;23:611-617. https://doi. org/10.1007/s11325-019-01799-2.

32. Watling CN, Mahmudul Hasan M, Larue GS. Sensitivity and specificity of the driver sleepiness detection methods using physiological signals: a systematic review. Accid Anal Prev 2021;150:105900. https://doi. org/10.1016/j.aap.2020.105900.

33. Colquhoun CP, Casolin A. Impact of rail medical standard on obstructive sleep apnoea prevalence. Occup Med (Lond) 2016;66:62-68. https:// doi.org/10.1093/occmed/kqv101.

34. Easterbrook MA, MacLean AW, Knowles JB. The prediction of performance by subjective measures and the MSLT. Sleep Res 1994;23:409.

35. Bhat A, Marciarille AM, Stevens D, Ingram DG. Drowsy driving considerations in non-commercial drivers for the sleep physician. J Clin Sleep Med 2019;15:1069-1071. https://doi.org/10.5664/jcsm.7898.

36. Wolkow AP, Rajaratnam SMW, Anderson C, Howard ME, Mansfield D. Recommendations for current and future countermeasures against sleep disorders and sleep loss to improve road safety in Australia. Intern Med J 2019;49:1181-1184. https://doi.org/10.1111/imj.14423.

37. Narcolepsy Network. Narcolepsy and driving laws [Internet]. Lynnwood, WA: Narcolepsy Network [cited 2021 Nov 28]. Available from: https:// narcolepsynetwork.org/narcolepsydrivinglaws/.
38. Government of British Columbia. 18 - Sleep disorders - CCMTA medical standards [Internet]. Victoria, BC: The Government of British Colombia [cited 2021 Nov 28]. Available from: https://www2.gov.bc.ca/ gov/content/transportation/driving-and-cycling/roadsafetybc/medical-fitness/medical-prof/med-standards/18-sleep-disorders.

39. Government of United Kingdom. Narcolepsy and driving [Internet]. London; Government of United Kingdom [cited 2021 Nov 28]. Available from: https://www.gov.uk/narcolepsy-and-driving.

40. Narcolepsy UK. Narcolepsy and driving [Internet]. Cambridgeshire: Narcolepsy UK [cited 2021 Nov 28]. Available from: https://www.narcolepsy.org.uk/resources/narcolepsy-and-driving.

41. Government of Netherland. Regulation requirements for suitability 2000 [Internet]. Government of Netherlands [cited 2021 Nov 28]. Available from: https://wetten.overheid.nl/BWBR0011362/2021-07-01.

42. Austroads. Assessing fitness to drive [Internet]. Sydney: Austroads [cited 2021 Nov 28]. Available from: https://austroads.com.au/publications/assessing-fitness-to-drive/ap-g56/sleep-disorders/general-assessment-and-managemenwl78b3ms/narcolepsy14.

43. New Zealand Transport Agency. Medical requirements for driving [Internet]. Wellington: Government of New Zealand [cited 2021 Nov 28]. Available from: https://www.nzta.govt.nz/driver-licences/gettinga-licence/medical-requirements/medical-reviews-and-revocations.

44. Bonsignore MR, Randerath W, Schiza S, et al. European Respiratory Society statement on sleep apnoea, sleepiness and driving risk. Eur Respir J 2021;57:2001272. https://doi.org/10.1183/13993003.01272-2020. 
Private/ Requirement

commercial for doctors separated to report

USA

California No Yes

Yes

South Dakoda No No No

Utah No No

Maryland No No

Maine

If a patient continue to drive against doctor's ad
Several levels of restriction that may be applied to drivers with narcolepsy.

Medical probation II: condition has been well controlled for 3 to 5 months.

Medical Probation type III: drivers with at least 6 months of control over symptoms, but are still at risk of experiencing sleepiness and/or cataplexy.

-Drivers with more than 6 months of control over symptoms may have restrictions lifted entirely.

Driving privileges can also be suspended if the doctor reports that the condition is not well controlled.

The driver's license application asks an applicant whether he or she in the past 12 months has experienced any narcoleptic episodes. Applicants who respond in the affirmative to these questions must have their physician complete a medical statement form.

Individuals who apply for or hold a license and have, or develop, or suspect physical, mental, or emotional impairment that may affect driving safety are responsible for reporting this to the division or its agent. "DO YOU HAVE OR HAVE YOU HAD, ANY OF THE FOLLOWING CONDITIONS IN THE LAST FIVE YEARS?” Do you have a condition that produces abnormal sleepiness (sleep apnea, narcolepsy, etc.?)

Driver's License Applicants are asked if they have a physical or mental condition that could affect driving. If the answer is yes, the applicant must have a physician complete medical evaluation form and they must complete a health questionnaire. Narcolepsy is on the list of conditions MD specifically asks about. Applications with medical evaluation forms are subject to evaluation by the state medical advisory board.

Maine specifically asks about Narcolepsy and requires a medical evaluation. There is a specific evaluation form for the doctor to fill out.

$<$ Non-commercial drivers $>$ eligible for a licence if:

- There have been no daytime sleep attacks or cataplexy, with or without treatment, during the past 12 months.

$<$ Commercial drivers > generally not eligible for a licence. May be eligible if:

- Sleep specialist supports driving commercial vehicles

- There have been no daytime sleep attacks or cataplexy during the last 12 months

Do not drive commercial vehicle for long hours, overnight or on irregular shifts

Not particularly mention 'narcolepsy'.

Driving licences shall not be issued for drivers suffering from a serious neurological disease, unless the application is supported by authorised medical opinion.

$<$ Private driver $>$

Individuals with narcolepsy may be considered fit if, based on a specialist report, adequate treatment is provided for at least two consecutive months.

- The Epworth Sleeping Scale score must be less than 11.

The eligibility period is one year the first time.

$<$ Commercial driver $>$

Persons with narcolepsy are permanently unfit.

Not particularly mention 'narcolepsy'.

If a person has health problems that cause seizures or other conditions that may make driving a vehicle difficult, the person might not be issued a driver's license or might have it suspended.

More strict rules for commercial drivers.

If a driver has narcolepsy, he must discuss this situation with a physician.

When applying for the first driving licence, a person with narcolepsy need a medical certificate of driving ability.

$<$ Private driver $>$

Narcolepsy patients must inform the Driver and Vehicle Licensing Agency (DVLA) of their condition, so that DVLA can make the necessary investigations affect their

ability to drive safely, and patients will be permitted to drive only if DVLA and can reach an informed decision on whether to permit patients to drive.

$<$ Commercial driver $>$

It would be unusual for a person with narcolepsy to be issued with licence.
Not particularly mention 'narcolepsy'.

If a person has health problems that cause seizures or other conditions that may make driving a vehicle difficult, the person might not be issued a driver's license or might have it suspended.

$<$ Private driver $>$

- A conditional licence may be considered by the driver licensing authority subject to periodic review, taking into accoun information provided by a specialist in sleep disorders on the response to treatment. $<$ Commercial driver $>$

- A conditional licence may be considered by the driver licensing authority subject to at least annual review, taking into accoun information provided by a specialist in sleep disorders as to whether the following criteria are met:

- Cataplexy has not been a feature in the past; and

- Medication is taken regularly; and

- There has been an absence of symptoms for six months; and

- Normal sleep latency present on Maintenance of Wakefulness Test (on or off medication)

New Zealand Yes Yes $\quad<$ Private driver $>$

Individuals should not drive if they have narcolepsy that is likely to impair ability to drive safely.

Driving should stop on diagnosis until:

- There is a satisfactory response to treatment, and clearance by an appropriate specialist, or

- Individual does not suffer from the full range of symptoms, in particular unpredictable episodes of cataplexy

$<$ Commercial driver $>$

Individuals who have severe narcolepsy or narcolepsy with excessive sleepiness or cataplexy are considered unfit to drive commercial vehicle. https://narcolepsynetwork.org/narcolepsydrivinglaws/

https://www2.gov.bc.ca/gov/content/transportation/drivingand-cycling/roadsafetybc/medical-fitness/medical-prof/medstandards/18-sleep-disorders

https://eur-lex.europa.eu/legal-content/EN/TXT/?uri=CELEX\%3 A02006L0126-20201101

https://wetten.overheid.nl/BWBR0011362/2021-07-01

https://www.mensura.be/en/blog/mandatory-certificate-offitness-to-drive

https://www.ajokortti-info.fi/en/maintaining-driving-skills/ driving-health

https://www.gov.uk/narcolepsy-and-driving

https://www.narcolepsy.org.uk/resources/narcolepsy-and-driving

(2013-12-11/ japan-severe-punishment-for-drivers-who-have-seizures-incertain-traffic-accident-cases/

https://austroads.com.au/publications/assessing-fitness-to-drive/ ap-g56/sleep-disorders/general-assessment-and-managemenwl78b3ms/narcolepsy14

https://www.nzta.govt.nz/driver-licences/getting-a-licence/medical-requirements/medical-reviews-and-revocations/ 